\title{
ARACNOIDITIS CRÓNICA*
}

\author{
Luis Alberto Garzón MD**, Anderson Arley Mesa MD***
}

\section{Resumen}

La aracnoiditis se refiere al compromiso inflamatorio de uno, varios o todos los segmentos de la capa media de las meninges, es decir la aracnoides. Se encuentra asociada con meningitis, neoplasias, hemorragia subaracnoidea, enfermedades infecciosas como tuberculosis, procedimientos invasivos por el uso de medios de contraste tecales, colocación de analgésicos y anestésicos a nivel del raquis, quimioterápicos intratecales, procesos autoinmunes, afecciones ginecológicas e incluso procesos no invasivos. Como en la actualidad esta entidad no se considera entre los primeros diagnósticos en pacientes con clínica de dolor tipo ardor en región lumbar irradiado a miembros inferiores, incontinencia urinaria y disestesias en artejos, queremos resaltar la importancia de tener en cuenta esta patología como diagnóstico diferencial.

Palabras clave: aracnoiditis, procedimientos analgésicos, anestésicos

Abreviaturas: RMN, resonancia magnética nuclear; LCR, líquido cefalorraquídeo; TBC, tuberculosis.

\section{CHRONIC ARACHNOIDITIS}

\section{Abstract}

Arachnoiditis is a broad term denoting inflammation of one, several or all segments of the middle layer of the meninges known as the arachnoid mater. It is related to meningitis, neoplasia, subarachnoid hemorrhage, infectious diseases such as tuberculosis, invasive procedures using intrathecal injection of contrast media, epidural injection of analgesics and anesthetic agents, intrathecal chemotherapy, autoimmune processes, gynecological disorders and even non invasive procedures. As this disorder is nowadays not considered within the main diagnostic possibilities in patients who manifest burning back pain irradiated to the lower limbs, bladder dysfunction and dysethesia of the toes, we wish to highlight the importance of also considering arachnoiditis as a differential diagnosis.

Key words: arachnoiditis, analgesic procedures, anesthetics

Fecha recibido:agosto 14 de 2012 - Fecha aceptado:abril 3 de 2013

* Grupo de investigación Deorum Opus, semilleros de investigación Findolor. Servicio de Anestesiología, Hospital de San José, Bogotá DC, Colombia.
** Médico anestesiólogo, algesiólogo, paliativista. Jefe de la Unidad Clínica de Dolor y Cuidado Paliativo, Hospital de San José. Profesor Asistente, Fundación Universitaria de Ciencias de la Salud, Bogotá DC, Colombia.

*** Interno XII semestre, Facultad de Medicina, Fundación Universitaria de Ciencias de la Salud, Bogotá DC, Colombia. 


\section{Introducción}

La aracnoiditis es una patología inflamatoria progresiva en la que se pueden comprometer uno, varios o todos los segmentos de la aracnoides, afectándose la médula espinal, el cerebro o ambos. La etiología no es única, se deriva de múltiples factores, dentro de ellos los infecciosos como la TBC, procedimientos analgésicos y anestésicos cómo inyecciones intratecales o epidurales, medios de contraste usados en mielografía, como etiofendilato (myodil en el Reino Unido o pantopaque en EEUU)', sangre en el espacio intratecal, neuroirritantes, neurolíticos, o neurotóxicos, intervenciones en columna vertebral, corticoesteroides o trauma. ${ }^{2}$

Objetivos: describir la aracnoiditis, como entidad catastrófica, de alto impacto social individual y familiar, subdiagnosticada y por ende subvalorada, que pasa inadvertida en un amplio grupo de pacientes. Cuando aparece suele considerarse como complicación de su enfermedad de base y no se enfoca como un nuevo diagnóstico. Esta revisión de tema se enfoca en la orientación clínica para extremar medidas preventivas y de esta manera reducir su incidencia y prevalencia.

\section{Materiales y métodos}

Se realizó búsqueda sistemática de la literatura, utilizando operadores booleanos AND, OR y NOT, límites de referencias halladas desde enero de 1970 hasta marzo de 2012. Se buscó en la base de datos (PUBMED) empleando las palabras clave adhesive arachnoiditis y spinal arachnoiditis. Como resultado se encontraron 373 referencias cuya revisión permite clasificar las complicaciones de la aracnoiditis según la incidencia de la mayor a la más baja así:

- Aracnoiditis osificante.

- Aracnoiditis TBC.

- Aracnoiditis crónica adhesiva.

- Aracnoiditis secundaria a uso de medios de contraste.

- Aracnoiditis por anestesia neuroaxial.

- Aracnoiditis espinal.

- Aracnoiditis y síndrome de la espalda fallida.

\section{Historia}

El primer caso de aracnoiditis fue reportado en 1869 , por Charcot y Joffroy ${ }^{3}$, quienes describieron dos casos de atrofia muscular progresiva con lesiones en los fascículos anterior y lateral de la médula espinal. Dos fases en el cuadro clínico fueron descritas por Quinke, proliferativa y crónica. ${ }^{4}$ Horsely en 1909 realizó la descripción de los síntomas de la enfermedad y la denominó "meningitis crónica espinal". ${ }^{5}$ Konig en 1905 hizo pública la primera descripción del déficit neurológico después de un procedimiento de anestesia peridural. ${ }^{6}$ Medios de contraste a base de aceite como pantopaque, introducido por Ramsey en 1944 que adquirió bastante popularidad por la excelente visualización y baja absorción en la circulación ${ }^{7}$, fueron reportados en varios estudios como causantes de daño neurológico ${ }^{8.9}$, por lo que se hizo necesario explorar medios diferentes con base en agua como la metrizamina, asequible en $1973 .{ }^{10}$

\section{Anatomía}

Las meninges craneales se caracterizan por proteger al encéfalo, dar soporte para arterias, venas y senos venosos, así como cerrar el espacio subaracnoideo (leptomeníngeo) para que el LCR que se produce en los plexos coroideos al interior de los cuatro ventrículos cerebrales, penetre para brindarle nutrición y sostenimiento al encéfalo. Son tres capas que en su orden de afuera hacia adentro se identifican como duramadre, aracnoides y piamadre. Las dos últimas se desarrollan a partir del mesénquima que rodea el cerebro embrionario, en conjunto se denominan leptomeninges (del griego membranas finas) y crean un espacio real lleno de líquido, el espacio subaracnoideo. ${ }^{11}$

La aracnoides tiene fibroblastos, fibras colágenas y en menor cantidad elásticas. No se encuentra unida a la duramadre y es la presión de LCR quien la mantiene adherida. ${ }^{11}$ Corresponde a la capa media que es la más delgada y frágil de las tres, desprovista de inervación y vascularización. ${ }^{12}$ Forma en el ensanchamiento del espacio subaracnoideo el saco dural, debajo del cono medular, que contiene la cauda equina y se denomina cisterna lumbar. ${ }^{13}$ 


\section{Factores de riesgo}

Algunos autores han identificado ciertos factores de riesgo, que hacen a los pacientes más susceptibles de sufrir complicaciones ${ }^{14,15}$, como enfermedad neurológica previa, obesidad y parestesias.

\section{Genética}

Dada la multiplicidad de mutaciones probables en cualquier gen, son aquellas que influyen en el proceso de cicatrización en cualquiera de sus fases produciendo alteraciones en el colágeno las que pueden conducir a la formación de queloide, trastorno que se cree es de herencia autosómica dominante. ${ }^{16}$

\section{Fisiopatología}

La aracnoiditis está comprometida en el proceso inflamatorio propio de esta capa en la cual se desencadena la quimiotaxis, proliferación y reorganización celular (Figura 1), que lleva a daño en la región nerviosa adjunta por distintos mecanismos como la compresión, dada a partir de exudados, fibroblastos y la reorganización del colágeno, es decir el proceso de cicatrización óptimo en cada lesión, que finaliza en adherencias nerviosas o compromiso del saco tecal. ${ }^{17} \mathrm{El}$ flujo cons-
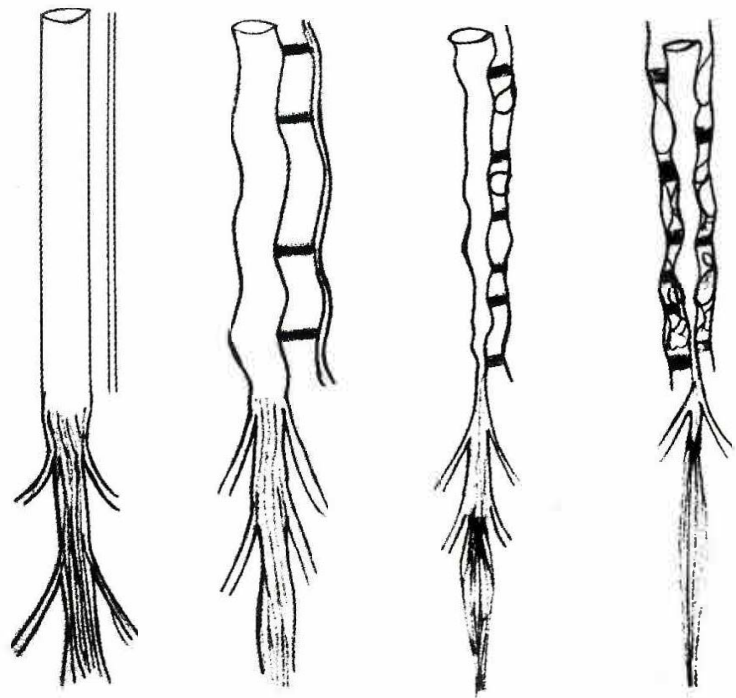

Figura I. Progresión de la aracnoiditis. tante del LCR también interfiere con el proceso de reorganización celular, dado que por la cinética constante del fluido que limpia o dispersa los fagocitos y las enzimas que previenen la formación de cicatriz. ${ }^{12}$

\section{Sintomatología}

No corresponde a un cuadro clínico patognomónico, aun cuando se describen síntomas de aparición frecuente (Tabla 1) y otros sugestivos hallados a través de la multiplicidad de estudios realizados desde Lombardi en 1961 con 41 pacientes, hasta Long en 1992 con 321 $\operatorname{casos}^{18-26}$ (Tabla 2) y que toman relevancia de acuerdo con los antecedentes del paciente.

\section{Tabla I. Síntomas de mayor prevalencia}

Dolor tipo ardor en la región lumbar irradiado a miembros inferiores.

Dolor persistente aún en reposo.

Poliuria.

Incontinencia urinaria.

Ardor en tobillos y pies.

Erupciones cutáneas y prurito inexplicado.

Parálisis parcial o completa de miembros inferiores.

Déficit neurológico.

Para establecer el grado de correlación entre la sospecha clínica y la imagenología se realizó en la última década un estudio norteamericano con una muestra retrospectiva de 436 pacientes llevados a la unidad de clínica del dolor a lo largo de catorce años por cuadro compatible con aracnoiditis. Se realizó examen neurológico completo y revisión de estudios solicitados durante su trayectoria clínica. Éstos pacientes fueron divididos en dos grupos, A y B, según presentaran o no déficit neurológico.

En el primero hubo 160 pacientes con déficit neurológico tras la realización de algún procedimiento neuroaxial, 11 de ellos tuvieron evidencia de proceso inflamatorio activo, 135 con disposición de raíces nerviosas en racimo y 12 con adhesión de raíces a saco dural; en el segundo, constituido por 276 pacientes, se hallaron raíces en racimo en 259, 152 con deformación del saco dural y en 241 fibrosis activa ${ }^{27}$ (Tabla 3). 


\section{Tabla 2. Hallazgos clínicos en la aracnoiditis crónica adhesiva}

\begin{tabular}{|c|c|c|c|c|c|}
\hline Lombardi ${ }^{18}$ & 1961 & 41 & $\begin{array}{l}\text { Dolor y parestesias } \\
\text { Anormalidad de esfinteres } \\
\text { Hipoestesia } \\
\text { Anormalidades del sistema motor }\end{array}$ & $\begin{array}{l}63 \\
63 \\
95 \\
98\end{array}$ & Mielografía \\
\hline De La Porte ${ }^{19}$ & 1973 & 38 & $\begin{array}{l}\text { Dolor de espalda } \\
\text { Dolor en miembros inferiores } \\
\text { Anormalidad de esfinteres } \\
\text { Reflejos anormales }\end{array}$ & $\begin{array}{l}50 \\
74 \\
29 \\
66\end{array}$ & Mielografía \\
\hline Benner ${ }^{21}$ & 1978 & 68 & $\begin{array}{l}\text { Dolor de espalda } \\
\text { Dolor en miembros inferiores } \\
\text { Déficit motor } \\
\text { Déficit sensitivo } \\
\text { Reflejos anormales } \\
\text { Incontinencia urinaria } \\
\text { Incontinencia fecal }\end{array}$ & $\begin{array}{l}84 \\
91 \\
72 \\
82 \\
88 \\
25 \\
16\end{array}$ & Mielografía \\
\hline Burton ${ }^{22}$ & 1978 & 100 & Dolor de espalda. Dolor de miembros inferiores & 100 & Mielografía \\
\hline Quiles ${ }^{23}$ & 1978 & 38 & $\begin{array}{l}\text { Dolor de espalda } \\
\text { Sintomas en miembros inferiores }\end{array}$ & $\begin{array}{l}76 \\
63\end{array}$ & Mielografía \\
\hline Long 26 & 1992 & 321 & $\begin{array}{l}\text { Dolor de espalda } \\
\text { Dolor de miembros inferiores } \\
\text { Claudicación neurogénica } \\
\text { Disminución de los movimientos del torso } \\
\text { Déficit motor } \\
\text { Déficit sensitivo } \\
\text { Reflejos anormales } \\
\text { Anormalidad esfinteriana } \\
\text { Progresión de los síntomas } \\
\text { Capacidad para caminar sin ayuda }\end{array}$ & $\begin{array}{l}94 \\
81 \\
92 \\
91 \\
74 \\
81 \\
96 \\
14 \\
18 \\
84\end{array}$ & Mielografía \\
\hline
\end{tabular}




\begin{tabular}{|c|c|c|c|}
\hline $\begin{array}{l}\text { Pacientes con } \\
\text { diagnóstico } \\
\text { clínico }\end{array}$ & Grupo & $\begin{array}{l}\text { Procedimiento } \\
\text { previo }\end{array}$ & $\begin{array}{l}\text { Pacientes-hallazgo } \\
\text { imagenológico }\end{array}$ \\
\hline \multirow[t]{2}{*}{436} & A- 160 & $\begin{array}{l}\text { Mielograma } \\
\text { Anestesia peridural } \\
\text { Anestesia raquidea } \\
\text { Parches epidurales } \\
\text { Inyecciones con } \\
\text { esteroides }\end{array}$ & $\begin{array}{l}\text { 11: inflamación de } \\
\text { raices nerviosas } \\
\text { 135: raíces en racimo } \\
\text { 12: adhesión de } \\
\text { raíces a saco tecal } \\
\text { 2: siringomielia }\end{array}$ \\
\hline & B-276 & $\begin{array}{l}\text { Intervención } \\
\text { quirúrgica del } \\
\text { raquis }\end{array}$ & $\begin{array}{l}\text { 259: raíces en racimo } \\
\text { 152: deformación de } \\
\text { saco dural } \\
\text { 241: fibrosis peridura }\end{array}$ \\
\hline
\end{tabular}

\section{Aracnoiditis osificante}

Es una variante crónica caracterizada por la osificación de la aracnoides de la médula espinal y se asocia con déficit neurológico progresivo. Se localiza con mayor frecuencia en la región sacra y en menor proporción en la torácica, su cuadro clínico surge por la mielopatía compresiva, dado por dolor lumbar bajo, debilidad en miembros inferiores y en ocasiones síndrome de compresión medular.

A nivel lumbosacro se manifiesta por dolor lumbar bajo y en miembros inferiores, cambios sensitivos, incontinencia urinaria, alteraciones intestinales y puede o no acompañarse con signos de radiculopatía. Aunque su patogénesis no está bien esclarecida, se piensa que es el estadío terminal de la aracnoiditis adhesiva relacionada con cirugía local previa, hemorragia subaracnoidea, mielografía, procesos infecciosos y anestesia espinal. ${ }^{28}$

Su diagnóstico se basa en la TAC multicorte, donde se aprecian calcificaciones alrededor de la médula espinal o el saco tecal y a nivel lumbosacro son intradurales, adyacentes a las raíces nerviosas. La RMN ha reemplazado cada vez más la TAC con y sin contraste tecal, los hallazgos en T2 son aglutinación de las raíces nerviosas de la cauda equina e imágenes hiper o hipointensas sin patrón fijo.

El curso y el pronóstico son variables, puede presentarse desde mielopatía compresiva con síntomas vagos, hasta osificaciones más grandes, ya que son factores independientes. El tratamiento está dirigido de acuerdo con la localización de la lesión y la presentación clínica. La microcirugía es útil para la extirpación de la calcificación, con la dificultad de que los resultados son pobres. Procedimientos como laminectomía descompresiva y foraminotomía, dan mejores resultados, aunque la terapia conservadora se sigue en el $50 \%$ de los casos. Es importante describir que el uso de esteroides no mejora el pronóstico de la enfermedad. ${ }^{28}$

\section{Aracnoiditis secundaria a uso de medios de contraste}

Para el diagnóstico de estenosis espinal de múltiples etiologías se utilizaron mielografías con medio de contraste como el isofendilate (myodil o pantopaque) y el lipiodol, es decir con base de aceite. El 1\% se complicaba con aracnoiditis por irritación e inflamación, en especial cuando se recolectaba el medio del espacio dural después del procedimiento. ${ }^{29}$

Estos medios se descontinuaron en los años 90 para dar paso a los provistos con base de agua, dentro de las cuales están la metrizamida y el iohexol. El cuadro clínico se manifiesta por dolor lumbar crónico, debilidad de miembros inferiores, incapacidad para la bipedestación autónoma, urgencia miccional y de deposición. ${ }^{30}$

\section{Aracnoiditis por TBC}

Enfermedad común en los países en vía de desarrollo cuya prevalencia va en ascenso. Dentro de la etiología infecciosa de la aracnoiditis es la más frecuente y a su vez con posibilidad de tratamiento. ${ }^{31}$ Dentro de las manifestaciones extrapulmonares el $10 \%$ corresponde al sistema nervioso central. Afecta el revestimiento aracnoideo a lo largo de toda la médula espinal, es una patología infrecuente que se manifiesta por debilidad en miembros inferiores, retención urinaria y puede cursar con déficit neurológico periférico progresivo.

Se describen tres teorías en la patogénesis de la aracnoiditis espinal por TBC: 1) lesión primaria en las meninges espinales, 2) extensión descendente de meningitis intracraneal por tuberculosis y extensión de 
la infección por espondilitis. La diseminación de la meningitis intracraneal es la teoría más aceptada y frecuente. La región torácica es muy afectada, seguida por la lumbar y cervical. En la macroscopía se observa exudado en la región periférica a la médula espinal y raíces nerviosas; al microscopio se observa inflamación granulomatosa con áreas de caseificación y tejido fibroso.

En 1996 el estudio mediante RMN con imágenes T1 de corte sagital y $\mathrm{T} 2$ en corte axial de la región afectada en 22 pacientes, quince hombres y siete mujeres entre 7 y 70 años, diagnosticados en la clínica como aracnoiditis espinal de etiología tuberculosa sin compromiso inmunológico, mostró lesiones nodulares en el espacio subaracnoideo, agrupamiento de las raíces nerviosas espinales y expansión de la médula espinal. ${ }^{31}$ Ocho de los diagnosticados con meningitis TBC desarrollaron mielopatía durante o después de la terapia con medicamentos, siendo la paraparesia el síntoma más común. En 16 de los 22 pacientes se presentaron manifestaciones como cuadriparesia, monoparesia, incontinencia urinaria, dolor lumbar y radiculopatía. La duración de los síntomas varió entre tres días y dos años; doce se asociaron con meningitis TBC, es decir cuatro nuevos hallazgos de meningitis intracraneal diagnosticada en la RMN por la presencia de exudados en las cisternas basales. ${ }^{31,32} \mathrm{El}$ análisis del líquido cerebroespinal presenta aumento de linfocitos, bajos niveles de glucosa y elevados de proteínas. ${ }^{32}$

El tratamiento es médico y/o quirúrgico. Para el primero se emplean isoniazida, rifampicina, pirazinamida y etambutol, hasta completar nueve a doce meses. Como tratamiento coadyuvante se usan corticoides como dexametasona vía oral en dosis de $16 \mathrm{mg} /$ día o vía intratecal durante tres a seis semanas. El tratamiento quirúrgico se basa en la realización de laminectomía según la compresión medular. ${ }^{33}$

\section{RMN en aracnoiditis espinal por TBC}

Mediante estudios realizados con el sistema $1.5 \mathrm{~T}$ Magnetom, Siemens, Ealangen, Alemania, y contraste con gadopentato $0.2 \mathrm{ml} / \mathrm{k}$, de 1993 a 1996 en 22 pacientes con diagnóstico clínico de aracnoiditis por TBC con edades comprendidas entre los siete y 70 años, revelaron los siguientes datos:

- El proceso inflamatorio abarca más de una región espinal; en nueve hubo compromiso de las regiones cervical y dorsal, en seis dorsal y lumbar, lumbosacra en tres casos, dos en la región dorsal y dos con extensión a todos los segmentos de la médula espinal.

- Las lesiones nodulares se localizaron en el espacio subaracnoideo de seis pacientes, de los cuales tres con compresión medular que se apreciaron bien en $\mathrm{T} 2$.

- Los cambios en la médula espinal vistos en 18 pacientes correspondieron a tres cavitaciones y doce expansiones de la médula espinal.

- La compresión extrínseca medular ocurrida en once casos, cinco con loculaciones, cuatro con lesiones nodulares en el espacio subaracnoideo y dos con absceso epidural.

- El engrosamiento y agrupamiento de raíces nerviosas se vio con frecuencia en los pacientes con aracnoiditis.

\section{Aracnolditis por anestesia neuroaxial}

La anestesia regional ha tenido gran auge en los últimos años, pero así mismo ha desencadenado algunas complicaciones tempranas como irritación de raíces nerviosas y de cauda equina y síndrome de cono medular, y tardías como radiculitis, agrupamiento de fibras nerviosas, fibrosis, cicatrices y deformidad del saco dural, paquimeningitis, pseudomeningocele y siringomielia.

En cuanto a la anestesia en el neuroaxis, la aracnoiditis ha causado abscesos epidurales. Para determinar si un procedimiento ocasionó un trauma, se deben evaluar síntomas como dolor tipo ardor en miembros inferiores, disestesia que no sigue el patrón de un dermatoma y disfunción sexual. Aparece una reacción local que está determinada por la dosis usada, el número de in- 
tentos en la punción, la solución solvente (solución salina normal, LCR), la respuesta inmune de cada paciente y el lugar de colocación del agente, sub, intra o peridural, que determinan la fase proliferativa dada por fibrosis, adhesiones y cicatrices ${ }^{34}$ (Tabla 4), denominada aracnoiditis porque es la capa del saco dural que contiene vesículas en la respuesta inmune. Estas mismas explican el fenómeno que se produce cuando hay sangre en el espacio subaracnoideo pues facilita el agrupamiento de fibras o raíces nerviosas en los bloqueos epidurales. ${ }^{35-37}$

\section{Tabla 4. Lesiones causantes de aracnoiditis}

Trauma en raices nerviosas.

Laceración de la vaina de mielina.

Herniación de axones.

Sangre en fluido cerebroespinal.

Colocación de neuroirritantes en espacio subaracnoideo.

Colocación de neurotóxicos en espacio epidural o subaracnoideo.

Es menos frecuente que se encuentre comprometida la piamadre, el tejido neural adyacente, la médula espinal, los ganglios, las fibras nerviosas y el cerebro. ${ }^{2} \mathrm{Se}$ proponen las siguientes formas de aracnoiditis:

- Irritación nerviosa temporal.

- Síndrome de cauda equina.

- Agrupamiento de raíces nerviosas.

- Adherencias de fibras nerviosas a la pared del saco tecal.

- Deformación del saco tecal.

- Pseudomeningocele.

- Calcificaciones intratecales.
- Paquimeningitis.

- Aracnoiditis osificante.

- Aracnoiditis optoquiasmática.

- Aracnoiditis cerebral.

La última se manifiesta por dolor facial intratable, cefalea aberrante, tics dolorosos y déficit visual y audiológico. ${ }^{38.39}$

\section{Aracnoiditis y síndrome de la espalda fallida}

Este síndrome se produce en aquellos pacientes en los que se han agotado todos los recursos para mejorar el dolor en espondilodistesis y pseudoartrosis. ${ }^{40-42}$ Suele presentarse por aracnoiditis después de tentativas de diagnóstico con mielogramas ${ }^{10,43,44}$, procedimientos invasivos que permiten la entrada de sangre al líquido cerebroespinal o intervenciones de neuromodulación..$^{45.46}$

\section{Diagnóstico radiológico}

Herramientas radiológicas como la TAC con medio de contraste y la RMN son muy útiles para el diagnóstico de aracnoiditis y facilitan su manejo y pronóstico ya que no causan irritación meníngea mayor a la establecida, como ocurre con métodos diagnósticos como la mielografía. La Tabla 5 muestra tres clasificaciones radiológicas que se han desarrollado a partir de la relación entre el proceso cicatricial y el saco dural con respecto a las raíces nerviosas. La primera se divide en leve y moderada, la segunda propuesta en 1995

\section{Tabla 5. Clasificación radiológica de aracnoiditis}

\begin{tabular}{|l|l|l|}
\hline \multicolumn{1}{|c|}{ I } & \multicolumn{1}{c|}{ II } & \multicolumn{1}{c|}{ III } \\
\hline $\begin{array}{l}\text { Leve: apariencia de saco caudal corto por adhesión } \\
\text { de fibras nerviosas dentro de las meninges. }\end{array}$ & $\begin{array}{l}\text { Grupo I leve: masa de raíces nervio- } \\
\text { sas centradas en el saco tecal. }\end{array}$ & $\begin{array}{l}\text { Tipo I: defecto unilateral, focal, de raíces } \\
\text { nerviosas que emergen junto al espacio in- } \\
\text { tervertebral. }\end{array}$ \\
\hline $\begin{array}{l}\text { Extensa: bloqueo parcial por la superficie irregular de } \\
\text { la cicatriz que aparenta acortamiento, estrechamien- } \\
\text { to y oclusión del saco tecal. }\end{array}$ & $\begin{array}{l}\text { Grupo II: raíces nerviosas adheridas } \\
\text { en la periferia de las meninges. }\end{array}$ & $\begin{array}{l}\text { Tipo II: defecto circunferencial o en forma } \\
\text { de anillo. }\end{array}$ \\
\hline & $\begin{array}{l}\text { Grupo III: masa de tejidos blandos que } \\
\text { remplaza el espacio subaracnoideo. }\end{array}$ & Tipo III:obstrucción transversa, completa. \\
\hline & & Tipo IV:pérdida de las estrías radiculares. \\
\hline
\end{tabular}


por Shiraishi ${ }^{47}$ considera tres tipos de aracnoiditis que van en orden ascendente según el compromiso del saco dural en la TAC, y la tercera hace referencia a los hallazgos de la mielografía que van desde una lesión unilateral a la salida de la raíz nerviosa a nivel vertebral, pasando por compresión anular de la raíz, hasta la pérdida de estrías radiculares, aspecto vítreo y alteración de la conducción nerviosa. Es así como el compromiso anatómico es proporcional al clínico y a la sintomatología presentada.

\section{Discusión}

La aracnoiditis crónica se presenta en los pacientes que han desarrollado un proceso inflamatorio previo sin tratamiento médico adecuado. Su diagnóstico no es fácil por el cuadro tan variado como síndrome de cauda equina, agrupamiento de raíces nerviosas, adherencias de fibras nerviosas a la pared del saco tecal, deformidad del mismo, pseudomeningocele, calcificaciones intratecales y osificación de la misma aracnoides. El aumento progresivo de la anestesia regional ha desencadenado algunas complicaciones que pueden ser tempranas o tardías, como irritación de raíces nerviosas, radiculitis, agrupamiento de fibras nerviosas, fibrosis y cicatrices.

Las opciones de manejo son médica o quirúrgica según sea la etiología. Cuando se trata de un proceso secundario a una patología se inicia tan pronto se confirma el diagnóstico. ¿De qué dependen entonces las posibilidades terapéuticas que se le pueden brindar a un paciente? De su presentación, del tiempo de inicio, estado inmunológico del paciente y soporte social y familiar. Aunque no hay un tiempo límite para relacionar ésta patología con procedimientos quirúrgicos previos, debe ahondarse en la historia clínica para investigar este antecedente.

\section{Conclusiones}

- Existe potencial amenaza de aracnoiditis al realizar la inserción de un catéter epidural o intratecal en la columna vertebral de aquellos pacientes que están recibiendo o van a recibir en un futuro terapia con anticoagulantes. ${ }^{40,48}$
- La aracnoiditis es una entidad que hay que tener en cuenta dentro de los diagnósticos diferenciales, ya que su presentación clínica es variada.

- Debe considerarse esta patología como de difícil manejo y por ende de pronta remisión para mejorar la calidad de vida del paciente.

- Los hallazgos radiológicos únicos de raíces en racimo, sugieren aracnoiditis por intervencionismo. ${ }^{27.49}$

- La aracnoiditis con antecedente quirúrgico a nivel del raquis presenta en los estudios radiológicos anormalidad del saco dural, cicatrizal y raíces en racimo. ${ }^{27}$

- La aracnoiditis asociada con deformidad anatómica de la columna, origina el cuadro clínico a partir del nivel comprometido. ${ }^{50}$

\section{Referencias}

1. Rice 1, Wee MY, Thomson K.Obstetric epidurals and chronic adhesive Arachnoiditis.Br J Anaesth. 2004 Jan;92(1):109-20.

2. Aldrete JA. Neurologic deficits and arachnoiditis following neuroaxial anesthesia. Acta Anaesthesiol Scand. 2003 Jan;47(1):3-12.

3. Charcot JM. Joffroy A. Deux cas d atrophie musculaire progressive avec lesions de la substance gris et des faisceaux anterolateraux de la moelle spinaire. Arch Physiologiei.1969;2: 354.

4. Horrax G. Generalized cisternal arachnoiditis simulating cerebellar tumor: its surgical treatment and end results. Arch Surg. 1924;9: 95-7.

5. Horseley V. Chronic spinal meningitis: its differential diagnosis and surgical treatment. Br J Med. 1909;1: 513-17.

6. Koning F. Bleiben de Rücken mark slähmngnach lumbal änesthesie. Munch Med Wchnschr.1906;53: 1112

7. Ramsey GH, French JD, Strain WH. Iodinated organic compounds as contrast media: for radiographic diagnosis. IV. Pantopaque myelography. Radiology. 1944:43: 236-40

8. Peacher WG, Peterson RCL. Pantopaque myelography: Results comparison of contrast media and spinal fluid reactions. J Neurosurgery. 1945:2: 220-31.

9. Tarlov IM. Pantopaque meningitis disclosed at operation. JAMA.1945; 128: 1014-16.

10. Metrizamide, a non-ionic, water-soluble contrast medium. Experimental and preliminary clinical investigations. Acta Radiol Suppl.1973;14: 1-387.

11. Moore KL, Dalley AF. Anatomía con orientación clínica. 4a ed. Madrid: Médica Panamericana; 2002. p. 892, 902-904.

12. Delamarter R, Ross J, Masaryk T, Modic M and Bohlman H. Diagnosis of lumbar arachnoiditis by magnetic resonance imaging. Spine. 1990; 15: 4304-10.

13. Moore KL, Dalley AF. Anatomía con orientación clínica. 4a ed. Madrid: Médica Panamericana; 2002. p. 491

14. Cheney FW, Dominio KB, Caplan RA, Pozner KL. Nerve injury associated with anesthesia. Anesthesiology.1999;90: 1060-69

15. Aldrete JA. Neurologic déficits and arachnoiditis following neuroaxial anesthesia. Acta Anaesthesiol Scand. 2003 Jan;47(1):3-12. 
16. ShikataJ, Yamamuro T. Lida H, and Sugimoto M. Surgical treatment for symptomatic spinal adhesive arachnoiditis. Spine. 1989; 14: 870-75

17. Toribatake Y, Baba H. Maezawa Y, Umeda S. and Tomita K. Symptomatic arachnoiditis ossilicans of the thoracic spine. Case report. Paraplegia. 1995 Apr:33(4):224-7.

18. Lombardi G, Passerini A, Migliavacca F. Spinal arachnoiditis. Br J Radiol. 1962; 35: $314-20$

19. De La Porte C, Seigfried J. Lumbosacral spinal fibrosis (Spinal arachnoiditis). Its diagnosis and treatment by spinal cord stimulation. Spine. 1983; 8: 593-603

20. Jorgensen J, Hansen V, Steenskov V. Ovesen N. A clinical and radiological study of chronic lower spinal arachnoiditis. Neuroradiology. 1975; 9: 139-44

21. Benner B. Ehni G. Spinal arachnoiditis. The postoperative variety in particular Spine. 1978; 3: 40-4

22. Burton C. Lumbosacral arachnoiditis. Spine. 1978; 3: 24-30

23. Quiles M, Marchisello P, Tsairis P. Lumbar adhesive arachnoiditis. Etiologic and pathologic aspects. Spine. 1978; 3: 45-50.

24. Shaw M. Russell JA, Grossart $\mathrm{KW}$. The changing pattern of spinal arachnoiditis J Neurol Neurosurg Psych. 1978; 41: 97-107

25. Guyer DW, Wiltse LL, Eskay ML, Guyer BH. The long-range prognosis of arachnoiditis. Spine. 1989; 14: 1332-41

26. Long DM. Chronic adhesive spinal arachnoiditis: pathogenesis, prognosis and treatment. Neurosurg Q. 1992; 2: 296-319

27. Aldrete JA, Ghaly RF. Brown TL. Vascello LA. et al.Correlación de hallazgos radiológicos con los eventos adversos que posiblemente hayan causado aracnoiditis.Rev. Soc. Esp. Dolor. 2005; 12 : 269-76

28. Chaun CC, Lau PY, Sun LK, Lo SS.Arachnoiditis ossificans.Hong Kong Med J. $2009 ; 15: 146-8$

29. Roca J, Moreta D, Ubierna M, Caceres E and Gomez JC. The results of surgical treatment of lumbar Arachnoiditis.Int Orthop. 1993;17(2):77-81.

30. Gnanalingham KK, Joshi SM, Sabin I. Thoracic arachnoiditis, arachnoid cyst and syrinx formation secondary to myelography with Myodil, 30 years previously. Eur Spine J. 2006 Oct:1 5 Suppl 5:661-3.

31. Sharma A, Goyal M, Mishra NK, Gupta V, Gaikwad SB. MR imaging of tubercular spinal arachnoiditis. AJR Am J Roentgenol.1997; 168(3):807-12.

32. de La Blanchardi'ere A, Stern JB, Molina JM, Lesprit P, Gasnault J, de Kerviler E, et al. Spinal tuberculosis arachnoiditis. Presse Med. 1996; 25(29): 1333-35.

33. Poon TL, Ho WS, Pang KY, Wong CK. Tuberculous meningitis with spinal tuberculous arachnoiditis.Hong Kong Med J. 2003; 9(1):59-61
34. Aldrete JA. Anatomopathology. In: Aldrete JA, editor. Arachnoiditis:the silent epidemic. Denver: FutureMed; 2000. p. 7-18.

35. Jourdan $\mathrm{C}$, Arthur F, Convert $\mathrm{J}$ et al. A rare and severe complication of meningeal hemorrhage: spinal arachnoiditis with paraplegia. Aggressiologie.1990; 31 : 413-4.

36. Tjandra JJ, Verma TR, Week RD. Spinal arachnoiditis following subarachnoid hemorrhage. Aust N Z Surg. 1989; 59: 884- 7

37. AldreteJA. Blood in the intrathecal space. In: Aldrete JA, editor. Arachnoiditis:the silent epidemic. Denver: FutureMed; 2000. p. 65-76.

38. Carrizo A. Optochiasmatic arachnoiditis. In: Aldrete JA, editor. Arachnoiditis:the silent epidemic. Denver: FutureMed; 2000. p.159-74

39. Aldrete JA. Cerebral arachnoiditis. . In: Aldrete JA, editor. Arachnoiditis:the silent epidemic. Denver: FutureMed; 2000. p. 175-8.

40. Sternlo JE, Hylebinette $\mathrm{CH}$. Spinal subdural bleeding after attempted epidural and subsequent spinal anesthesia in a patient with thormboprophylaxis with low molecular weight heparin. Acta Anaesthsiol Scand. 1995; 39: 557-9.

41. Burton CV. Causes of failure of surgery in the lumbar spina: 10-year follow-up. Mt Sinai Med J.1991; 2: 58-65.

42. Turner JA, Ersek M, Herron L et al. Patient outcomes after lumbar spinal fusion: a comprehensive literature synthesis. JAMA. 1992; 268: 907-I 1.

43. Howland WJ, Curry JL, Butler AK. Pantopaque arachnoiditis: experimental study of blood as potentiating agent. Radiology.1963; 80: 371-79.

44. Hansen EB, Fahrenkrug A, Praestholm J. Late meningeal effects of myelographic contrast media with special referenceto metrizamide. Br J Radiol. 1978; 51 : 321-77.

45. Aldrete JA, Zapata JC, Ghaly RF. Arachnoiditis following epidural adhesiolysis in chronic failed back surgery patients. Clin J Pain.1995; 11 : 147-50.

46. Deyo RA. Practice variations, treatment fads, rising disability. Do we need a clinical research paradigm?.Spine.1993; 18: 2153-62.

47. ShiraishiT, Crock HV, Reynolds A. Spinal arachnoiditis ossificans. Observations on its investigation and treatment. Eur Spine J. 1995;4(1):60-3.

48. Horlocker TT, Wedel DJ. Neuroaxial block and low molecular weight heparin. balancing perioperative analgesia and thromboprophylaxis. Acta Anaesthesiol Scand.1995; 39: 557- 9.

49. North RB, Campbell JN, Javers CS et al. Failed back surgery syndrome: a 5-year follow-up in 102 patients undergo in grepeated operations. Neurosurgery.1991; 28: $685-91$.

50. Boume IH. Lumbo-sacral adhesive arachnoiditis: A review. J R Soc Med. 1990 Apr;83(4):262-5. 